\title{
DESCENTRALIZAÇÃO DO PROGRAMA SEGUNDO TEMPO E NÍVEIS DE DESENVOLVIMENTO HUMANO (IDH)
}

Recebido em: 05/08/2018

Aceito em: 09/04/2019

\author{
Edmilson Santos dos Santos ${ }^{1}$ \\ Universidade Federal do Vale do São Francisco (UNIVASF) \\ Petrolina - PE - Brasil
}

RESUMO: O Programa Segundo Tempo do Ministério do Esporte procura democratizar o acesso ao esporte educacional via descentralização de suas ações (topdown). Neste sentido, este trabalho descritivo-exploratório analisou o resultado da alocação de recursos do governo federal na descentralização do Programa Segundo Tempo aos governos municipais em 2003, ano de implantação do programa, até 2012. As informações buscaram medir a frequência de implementação do programa em conjunto com os municípios e associá-los ao Índice de Desenvolvimento Humano (IDH). Os dados mostraram que os incentivos para a descentralização não foram adequados às necessidades de implementação do programa com os municípios mais vulneráveis e que os municípios menos vulneráveis apresentaram melhores condições institucionais para sua difusão.

PALAVRAS CHAVE: Esportes. Atividades de Lazer. Políticas Públicas.

\section{DECENTRALIZATION OF THE PROGRAM SEGUNDO TEMPO AND LEVELS OF HUMAN DEVELOPMENT (HDI)}

ABSTRACT: Program Segundo Tempo, from the Ministry of Sports, seeks to democratize access to educational sport through decentralization of its own actions (topdown). In this sense, this descriptive-exploratory work analyzed the result of the federal government's allocation of resources in the decentralization of the Program Segundo Tempo to municipal governments in 2003, the year of implementation of the program, until 2012. The information sought to measure the frequency of implementation of the program in with the municipalities and associate them with the Human Development Index (HDI). The data showed that the incentives for decentralization were not adequate to the implementation needs of the program with the most vulnerable municipalities and that the less vulnerable municipalities presented better institutional conditions for their diffusion.

\footnotetext{
1 Professor do Colegiado de Educação Física da Universidade Federal do Vale do São Francisco. Pesquisador da área de políticas públicas de esporte e de lazer. Coordenador do Centro de Desenvolvimento de Políticas Públicas de Esporte e de Lazer do Piauí.
} 


\section{KEYWORDS: Sports. Leisure Activities. Public Policy.}

\section{Introdução}

A expressão vulnerabilidade, como forma de apontar o risco social, possui uma força discursiva muito potente do ponto de vista político, capaz de produzir agenda, apesar de apresentar muitas fragilidades ou limitações quando a transportamos para o campo empírico. Há um consenso no debate político de que este problema é capaz de indicar, no final do processo de representação, indivíduos ou grupos que devem obter uma atenção especial por parte do Estado. Portanto, compreender esse cenário torna-se útil para desenvolvermos reflexões que possam dar maior consistência á análise da política pública.

Esse tema passou a pautar o debate acadêmico brasileiro, de uma forma mais intensa, a partir da década de noventa, dentro de um período de grande disputa em torno das políticas sociais no Brasil. Para além do que foi proposto pela agenda neoliberal, a focalização - a eficiência dos gastos públicos - significou a busca e a localização daquele portador de maiores dificuldades na garantia do seu bem-estar social. Ser vulnerável ou encontrar-se em situação de risco social, entendidos aqui como sinônimos, era a senha para a implementação de políticas públicas nas mais diferentes áreas. Por conta disso, diferentes áreas passaram a fazer uma análise particular do que era possível ser interpretado como vulnerabilidade e ela passa a ser elemento auxiliar para a análise da realidade social.

Em linhas gerais, a vulnerabilidade é identificada em situações ou contextos em que grupos, famílias ou indivíduos encontram-se em condições de desvantagem, marginalidade ou exclusão (BUSSO, 2001). Dentro de um gradiente de dificuldades, vulnerável é aquele que se encontra na ponta extrema do processo de exclusão e que 
passa a receber atenção por parte do Estado. Nesse sentido, o processo de significação da vulnerabilidade também é resultado de uma disputa política que busca não apenas conhecê-la, mas também definir o papel do Estado na superação desse problema: do acolhimento à exclusão espacial (segregação ou prisão) ou à exclusão física (eliminação).

A vulnerabilidade pode ser definida a partir de critérios econômicos, como a renda per capita (como o Programa Bolsa Família); através de critérios espaciais, ao ser morador de uma determinada região que sofre com o acesso de determinados serviços; como resultado da violência urbana, ou da pobreza. Na página inicial do Programa Segundo Tempo no site do Ministério dos Esportes aparece uma preocupação espacial da vulnerabilidade: áreas de vulnerabilidade social. Princípio em consonância com o que está previsto como atribuição da União em nossa constituição no inciso $3^{\circ}$ do artigo $3^{\text {o }}$ da Constituição Federal de 1988. Nossa federação aponta como missão do Estado reduzir assimetrias alocativas (PAES; SIQUEIRA, 2008; ARRETCHE, 2004; MENDES, 2012).

Por vulnerabilidade costuma-se designar uma variedade de fenômenos sociais que buscam demarcar uma desvantagem vivida. Em muitos casos, há um reconhecimento de que essa desvantagem produz uma desigualdade no acesso ao bemestar social. Se as famílias ou os indivíduos não possuem condições de superar essa dificuldade, seja junto a sua comunidade ou no mercado, cabe ao Estado atuar de forma a diminuir sua vulnerabilidade.

Dada a complexidade dos fenômenos sociais, a vulnerabilidade não é uma expressão aglutinadora e unificadora de todas as dificuldades por que possam estar passando determinados indivíduos e comunidades. As vulnerabilidades são múltiplas, e 
o número de exposições a determinados eventos aumenta a fragilidade desses indivíduos. Podemos encontrar situações de desvantagens, portanto vulnerabilidade, inclusive no esporte e no lazer como demonstrou o Perfil dos Municípios do IBGE (IBGE, 2006, 2017). Não é sem razão que o Programa Segundo Tempo (PST) do Ministério do Esporte indica a vulnerabilidade como um marcador importante de sua política.

O PST foi criado de forma incremental pelo Ministério do Esporte no primeiro ano do Governo de Lula. Ele surge a partir dos programas Esporte na Escola e Esporte solidário (FERREIRA; CASSIOLATO; GONZALEZ, 2009). O primeiro buscava alcançar a prática esportiva aos alunos de escolas públicas. O segundo estava direcionado à garantia do acesso a atividade esportiva às comunidades carentes. Surge, portanto, com uma forte preocupação de combate a exclusão social, política prioritária na gestão do Governo Lula (ALMEIDA, 2004), e garantir atenção à criança no turno inverso. Apesar da preocupação com o turno inverso, o programa nasce desvinculado de uma política mais ampla de educação integral.

Programas descentralizados por oferta, como é o caso do PST, exigem o desejo de cooperação dos entes subnacionais, governos estaduais e municipais, e de parceiros da sociedade civil, como o terceiro setor. A federação brasileira não suporta imposições de cima para baixo fora aquelas previstas na Constituição Federal de 1988. Os agentes descentralizadores deverão demonstrar interesse em implementar o programa.

Nesse sentido, as estratégias elencadas para efetivação do PST não estão desvinculadas da sua natureza. A implementação produz a política (HILL, 2006). Não é uma parte outra que deve ser analisada separadamente. A política é aquilo que ela produz de resultado e não o ideário desenhado nos documentos do programa. 
A não cooperação não implica na subtração de oferta de determinada política pública, pois o esporte educacional, foco principal do programa, poderá ser ofertado por qualquer uma das instituições com base na autonomia decisória de cada um dos entes federados e do terceiro setor (apoiado pelo estado ou pela iniciativa privada). Nenhum agende descentralizador está refém da agenda do Governo Federal.

No entanto, é senso comum de que faltam recursos para garantir o acesso a programas e projetos sociais destinados ao esporte e ao lazer (mesmo que nos faltem parâmetros para essas avaliações). No entanto, a iniciativa do Governo Federal acrescenta recursos no sistema e eles deveriam ser empregados a fim de garantir o princípio da equidade.

Apesar dos constituintes terem garantido o direito ao esporte inscrito no artigo 217 da $\mathrm{CF} / 88$, ele não ofertou ferramentas necessárias ao enfrentamento dessa questão por parte da federação. Nos artigos que tratam das competências exclusivas e comuns do executivo dos três entes federados, União, estados/Distrito Federal e municípios), não foi imposta nenhuma obrigatoriedade à implementação de políticas de esporte e de lazer. Essa missão cabe aos legislativos (inciso $9^{\circ}$ do artigo 24 da $\mathrm{CF} / 88$ ). Importante salientar que qualquer obrigação a ser referendada ao poder executivo necessita garantir o princípio da legalidade (artigo 37 da $\mathrm{CF} / 88$ ). Nesse ponto reside um trade-off entre o direito do cidadão e o dever do governo de implementar políticas de esporte e de lazer.

O desenho do programa, a forma como foi pensado a adesão das prefeituras, possui incentivos que podem privilegiar determinados atores e dificultar a adesão de 
outros $^{2}$. O sucesso de programas descentralizados por oferta, que exigem adesões voluntárias, não pode desconsiderar elementos essenciais à adesão [como a desigualdade, a capacidade técnica dos parceiros, os incentivos ofertados à cooperação e a relação custo-benefício (MACHADO, 2008; BONFIM; SILVA, 2003; SOUZA, 2001)] e promover desigualdade (ARRETCHE; VAZQUEZ; GOMES, 2012; ARRETCHE; MARQUES, 2007; MELO, 2005). Por outro lado, o ator privilegiado pela CF/88 para diminuir desigualdades é o Governo Federal, a quem cabe a construção de políticas que promovam a justiça social (MARQUES; ARRETCHE, 2003).

Diante do fato de que os estudos sobre o PST não têm explorado de forma comparativa o saldo de sua implementação no território nacional por níveis de Índice de Desenvolvimento Humano (IDH), a pergunta que norteou a presente investigação busca preencher essa lacuna: A descentralização do PST pelas prefeituras (descentralização top-down $)^{3}$ alcançou resultados redistributivos nos 10 primeiros anos de sua implementação?

Sendo assim, o presente trabalho objetivou verificar se a descentralização do Programa Segundo Tempo realizada pelas prefeituras alcançou de forma prioritária os municípios mais vulneráveis, alcançando o que está previsto no inciso 3 do artigo 3 da Constituição Federal de 1988 (BRASIL, 1988).

As hipóteses de trabalho estão associadas aos critérios de análise:

\footnotetext{
${ }^{2}$ A implementação do PST até 2008 seguiu a Instrução Normativa $n^{\circ}$ 01, de 15 de janeiro de 1997 (BRASIL, 1997) e após a Portaria Interministerial $n^{\circ}$ 127, de 29 de maio de 2008 (BRASIL, 2008). Os dois instrumentos jurídicos não impunham restrições à focalização. Em 2011 a Secretaria Nacional de Esporte, Lazer e Inclusão Social lança Edital para seleção de propostas. Em todo o período o Ministério do Esporte cumpriu um papel estratégico e constitucional de estabelecer critérios e selecionar propostas. Portanto, o Ministério do Esporte foi um ator ativo para o resultado alcançado pelo programa.

3 A descentralização top-down acontece quando um ente subnacional, podendo ser a prefeitura ou o governo estadual, decide implementar uma ação formulada pelo ente federado superior. No caso em pauta a União. Ela difere da descentralização bottom-up que acontece quando o ente descentralizado, no caso as prefeituras, decidem formular e financiar um programa para uma determinada área (SABATIER, 1986).
} 
H1: A descentralização do PST produziu efeito redistributivo e privilegiou os municípios mais vulneráveis;

H2: A descentralização do PST reproduziu a desigualdade da distribuição dos níveis de IDH;

H3: A descentralização do PST promoveu mais desigualdade privilegiando os municípios menos vulneráveis.

\section{Metodologia}

O presente trabalho de natureza descritivo-exploratório analisou o resultado da alocação dos recursos do governo federal na descentralização do Programa Segundo Tempo às prefeituras de 2003, ano de implantação do programa, a 2012. As informações buscaram aferir a frequência de implementação do programa junto aos municípios e associá-los ao Índice de Desenvolvimento Humano (IDH).

Utilizamos como critério para análise da implementação do programa subótima, ótima e conservadora:

(a) Compreendemos como implementação subótima quando o percentual de implementação do programa se equipara à distribuição percentual dos municípios por nível de desenvolvimento humano aferido pelo Índice de Desenvolvimento Humano Municipal (2010). Considerou-se como margem de erro 1 ponto percentual (pp) para cima ou para baixo. Ou seja, o programa foi implementado de forma semelhante ao peso dos níveis de IDH na realidade nacional.

(b) A implementação ótima se dá quando o percentual de implementação do programa favorece sua distribuição em direção aos municípios mais vulneráveis. Essa distorção indicará que variáveis importantes atuam de forma a priorizar as prefeituras mais vulneráveis do que aqueles menos vulneráveis. 
(c) A implementação conservadora se dará quando ocorrer o inverso da implementação ótima. Os municípios menos vulneráveis são priorizados na distribuição do programa.

O marcador vulnerabilidade foi definido a partir do IDH municipal/2010. O IDH foi dividido em cinco níveis: Muito Baixo [IDHMB (0 a 0,499)]; Baixo [IDHB (0,500 a 0,599)]; Médio [IDHM (0,600 a 0,699)]; Alto [IDHA $(0,700$ a 0,799)]; e Muito Alto [IDHMA (acima de 0,800)].

Para efeito deste trabalho, consideraremos como cidade vulnerável aquela que se encontra abaixo de 0,599 do IDH. Esse grupo se divide em outros dois: muito baixo, até 0,499, e baixo, entre 0,500 e 0,599. As informações sobre o Programa Segundo Tempo foram solicitadas diretamente ao Ministério do Esporte via Lei de Acesso à Informação (Protocolo 58750000323201676). A relação de municípios foi extraída diretamente do site do Instituto Brasileiro de Geografia e Estatística (IBGE). Considerou-se o IDH de 2010 fornecido diretamente no site do Programa das Nações Unidas para o Desenvolvimento.

A ideia do estudo foi trabalhar com o estoque de adesões ao logo dos 10 primeiros anos. Não foi realizada distinção entre as prefeituras que aderiram ao programa uma ou 10 vezes. Todos os municípios que implementaram o programa no intervalo de tempo aqui determinado compuseram a população do estudo.

O desenho metodológico seguiu a seguinte sequência: (a) constituição de banco de dados com todas as prefeituras brasileiras e suas regiões; (b) inclusão do IDH das cidades brasileiras; (c) inclusão de todas as cidades que tiveram a implementação do programa de 2003 a 2012. 


\section{Análise dos Resultados}

A desigualdade e a exclusão são fenômenos socioeconômicos produzido por um processo político multidimensional de elevada complexidade. Apesar de forças econômicas externas terem um papel importante na configuração dessa realidade, a intensidade da luta política em cada um dos municípios brasileiros e em cada uma das áreas de atuação podem produzir diferenças na forma de experimentação do quadro de desigualdade e exclusão. Como pode ser observado nas tabelas que seguem, esse quadro não nos permite pensar em uma realidade regional homogênea. Há proximidades e distensões importantes a serem observadas.

A primeira observação a fazer é que os níveis de IDH não se distribuem de forma equânime entre as regiões. As regiões Sul e Sudeste possuem seus maiores valores de distribuição percentual no IDH alto, 65,01\% e 51,74\%, respectivamente, enquanto que a região Nordeste concentra no IDH baixo, 60,59\% (como pode ser observado na Tabela 1). As regiões Norte e Centro-Oeste concentram sua maior distribuição dos municípios no nível de desenvolvimento médio. Outro fator de desequilíbrio é o percentual de municípios nos dois extremos da escala. As regiões Norte, Nordeste e Centro-Oeste não possuem municípios no nível mais alto de desenvolvimento humano. E as regiões Norte e Nordeste são as únicas que possuem municípios no nível mais baixo.

Considerando apenas a classificação de vulnerabilidade assumida neste trabalho (Tabela 1), IDH muito baixo e baixo, o cenário coloca a região Nordeste como a que possui o maior percentual de municípios vulneráveis, com $61,37 \%$, seguido da região Norte, 43,88\%. As regiões Sudeste, Sul e Centro-Oeste com percentual abaixo de 5\% 
$(4,74 \%, 0,59 \%$ e $3,23 \%$, respectivamente). A região Nordeste é a mais vulnerável pelo número de municípios, pois possui 78,7\% dos municípios vulneráveis.

Tabela 1: Distribuição dos municípios conforme níveis de IDH por região

\begin{tabular}{lcccccccccc}
\hline \multicolumn{1}{c}{ IDH } & \multicolumn{2}{c}{ Norte } & \multicolumn{2}{c}{ Nordeste } & \multicolumn{2}{c}{ Sudeste } & \multicolumn{2}{c}{ Sul } & \multicolumn{2}{c}{ Centro-Oeste } \\
\hline & $\mathrm{N}$ & $\mathbf{0}$ & $\mathrm{N}$ & $\mathbf{0}$ & $\mathrm{N}$ & $\mathbf{0}$ & $\mathrm{N}$ & $\mathbf{0}$ & $\mathrm{N}$ & $\%$ \\
\hline Muito & & & & & & & & & & \\
Baixo & 18 & 4 & 14 & 0,7 & 0 & 0 & 0 & 0 & 0 & 0 \\
Baixo & 179 & 39,8 & 1087 & 60,5 & 79 & 4,7 & 7 & 0,5 & 15 & 3,2 \\
Médio & 227 & 50,5 & 654 & 36,4 & 698 & 41,8 & 394 & 33,1 & 260 & 55,9 \\
Alto & 25 & 5,5 & 39 & 2,1 & 863 & 51,7 & 773 & 65 & 190 & 40,8 \\
Muito Alto & 0 & 0 & 0 & 0 & 28 & 1,6 & 15 & 1,2 & 0 & 0 \\
\hline Fonte: PNUD & & & & & & & & & &
\end{tabular}

Fonte: PNUD.

Apesar de não termos uma escala valorativa nem uma métrica para avaliar a capacidade de implementação do Programa Segundo Tempo e diante da autonomia política dos entes federados de aderirem ou não às iniciativas do Governo Federal, ter alcançado 8,9\% $(\mathrm{n}=495)$ das prefeituras em 10 anos de implementação demonstra baixa capacidade de descentralização junto a esses atores. Uma das motivações para esse comportamento é que as prefeituras podem ter seus próprios programas sociais na área esportiva. Ou, os incentivos dados a elas são inadequados.

A baixa adesão demonstra que não é uma política que veio para fortalecer as relações federativas brasileiras. Esse resultado pode demonstrar também baixo interesse pelo programa ou os incentivos à descentralização não serem atraentes. As restrições orçamentárias, tão bem detalhadas por Castro (2016), interferem na análise global, mas tem pouco impacto na seleção das prefeituras em si. Dado o baixo alcance do programa em termos de adesão de prefeituras, importante observar sua distribuição geográfica de forma a compreender o saldo líquido de sua implementação.

Tabela 2: Distribuição percentual das prefeituras que descentralizaram o PST por ano/região (\%).

\begin{tabular}{ccccccccccr}
\hline Região* & $\mathbf{2 0 0 3}$ & $\mathbf{2 0 0 4}$ & $\mathbf{2 0 0 5}$ & $\mathbf{2 0 0 6}$ & $\mathbf{2 0 0 7}$ & $\mathbf{2 0 0 8}$ & $\mathbf{2 0 0 9}$ & $\mathbf{2 0 1 0}$ & $\mathbf{2 0 1 1}$ & $\mathbf{2 0 1 2}$ \\
\hline S & 26,47 & 34,21 & 35,29 & 21,01 & 25,74 & 6,25 & 12,87 & 18,57 & 15,32 & 18,75 \\
SD & 23,53 & 26,32 & 22,55 & 41,18 & 31,68 & 62,5 & 59,41 & 40,00 & 43,24 & 62,5 \\
\hline
\end{tabular}




\begin{tabular}{crrrrrrrrrr}
\hline CO & 0 & 2,63 & 6,86 & 7,56 & 5,94 & 0 & 0,99 & 8,57 & 2,70 & 0 \\
ND & 35,29 & 34,21 & 32,35 & 24,37 & 30,69 & 25 & 23,76 & 31,43 & 36,94 & 18,75 \\
N & 14,71 & 2,63 & 2,94 & 5,88 & 5,94 & 6,25 & 2,97 & 1,43 & 1,80 & 0 \\
\hline
\end{tabular}

Fonte: Dados da pesquisa. * Sul (S); Sudeste (SD); Centro-Oeste (CO); Nordeste (ND); Norte (N)

O Governo Federal é o único ente federado com capacidade de diminuir diferenças alocativas (de recursos ou serviços) no âmbito federal. Por isso foi dado a ele a missão de diminuir as desigualdades regionais (inciso 3 do art. 3 da $\mathrm{CF} / 88$ ). Mesmo sabendo que há municípios vulneráveis também nas regiões mais desenvolvidas, como Sul e Sudeste, a maior carência de políticas sociais se dá nas regiões Nordeste e Norte. Portanto, elas deveriam ser priorizadas por um programa que almeja justiça social.

Considerada a premissa de que cabe ao governo federal diminuir desigualdades regionais, o resultado da descentralização do PST representa uma grande dificuldade política de sustentação do programa por dentro da estrutura federalista brasileira. O período em que teve menor distribuição percentual do programa em direção às regiões Sul e Sudeste foi em 2003. Nesse ano a descentralização para essas regiões foi de $50 \%$. Em 2012 esse valor alcançou 81,25\%, demonstrando de forma cabal que a implementação do programa serviu para ampliar as desigualdades existentes no Brasil no que diz respeito ao acesso às políticas públicas de esporte e de lazer. Ou seja, sob esse critério, o PST seguiu na contramão da diminuição da desigualdade.

Excetuando o primeiro ano em que as regiões Nordeste e Norte alcançaram 50\% da adesão ao programa, nos outros períodos a adesão variou entre 31\% a 38\%. Em 2012 a adesão ficou em 18,75\%. Isso significa que variáveis importantes atuaram e o programa não alcançou o que está previsto na constituição, diminuição das desigualdades regionais.

Quando analisamos a distribuição do PST por níveis de IDH observamos que o programa atuou mais uma vez no sentido de aprofundar as desigualdades. Primeiro, 
porque nenhuma prefeitura cujo município tem IDH Muito Baixo conseguiu descentralizar o programa nos 10 primeiros anos de implementação. Segundo, porque os municípios de IDH Baixo respondem apenas por 8,48\% da descentralização do programa pelas prefeituras. Isso significa que os municípios alvo prioritários do programa foram preteridos. Para corroborar com essa afirmação prévia, a adesão das prefeituras com municípios de IDH Muito Alto e Alto foi da ordem de 58,59\%. Ou seja, o desenho do programa não conseguiu imprimir uma força redistributiva e alcançar, de forma prioritária, as prefeituras dos municípios mais vulneráveis. Diferentemente do que aconteceu com os municípios menos vulneráveis.

A análise descritiva da Tabela 3 permite perceber que: (a) as regiões Sul e Sudeste, as mais desenvolvidas, ao lado da Região Centro-Oeste descentralizaram o PST para municípios de maior grau de desenvolvimento humano; (b) as regiões Sul e Centro-Oeste não descentralizaram o PST nem para os municípios de IDH Muito Baixo, nem para IDH Baixo; (c) em nenhum cenário a distribuição percentual dos municípios que receberam o PST foi o maior alcançado, indicando maior prioridade; (d) exceto o Nordeste, o PST privilegiou municípios de IDH Alto.

Tabela 3: Distribuição das prefeituras que descentralizaram o PST por níveis de IDH e região.

\begin{tabular}{lcccccccccc}
\hline \multicolumn{1}{c}{ IDH } & \multicolumn{2}{c}{ Norte } & \multicolumn{2}{c}{ Nordeste } & \multicolumn{2}{c}{ Sudeste } & \multicolumn{2}{c}{ Sul } & \multicolumn{2}{c}{ Centro-Oeste } \\
\hline & $\mathrm{N}$ & $\%$ & $\mathrm{~N}$ & $\%$ & $\mathrm{~N}$ & $\%$ & $\mathrm{~N}$ & $\%$ & $\mathrm{~N}$ & $\%$ \\
\hline Muito Baixo & 0 & 0 & 0 & 0 & 0 & 0 & 0 & 0 & 0 & 0 \\
Baixo & 3 & 16,6 & 38 & 26,3 & 1 & 0,4 & 0 & 0 & 0 & 0 \\
Médio & 7 & 38,8 & 90 & 62,5 & 39 & 18,5 & 21 & 21,6 & 6 & 23,0 \\
Alto & 8 & 44,4 & 16 & 11,1 & 151 & 71,9 & 71 & 73,2 & 19 & 73,0 \\
Muito Alto & 0 & 0 & 0 & 0, & 19 & 9 & 5 & 5,1 & 1 & 3,8 \\
\hline Fonte: PNUD & & & & & & & & & &
\end{tabular}

Fonte: PNUD.

Os resultados destacados na Tabela 3 indicam que o desenho do PST não consegue criar incentivos à descentralização que seduza os municípios mais vulneráveis a participarem do programa. Suas estratégias de seleção das propostas podem estar 
provocando distorção alocativa ao privilegiar municípios de maior desenvolvimento social em detrimento dos de maior vulnerabilidade.

Tabela 4: Diferença em pontos percentuais (pp) entre a distribuição do PST por

\begin{tabular}{lccccc}
\multicolumn{7}{c}{ região e níveis de IDH. } \\
\hline \multicolumn{1}{c}{ IDH } & Norte & Nordeste & Sudeste & Sul & Centro-oeste \\
\hline Muito Baixo & 4 & 0,7 & 0 & 0 & 0 \\
Baixo & 23,2 & 34,2 & 4,3 & 0,5 & 3,2 \\
Médio & 11,7 & $-26,1$ & 23,3 & 11,5 & 32,9 \\
Alto & $-38,9$ & -9 & $-20,2$ & $-8,2$ & $-32,2$ \\
Muito Alto & 0 & 0 & $-7,4$ & $-3,9$ & $-3,8$ \\
\hline
\end{tabular}

Fonte: Dados da pesquisa.

Seguindo os critérios aqui estabelecidos (margem de erro de 1 pp para nível subótimo) pode-se perceber que a diferença entre os níveis IDHMB, para a região Nordeste, e IDHB, para a região sul, demonstra que a distribuição percentual dos municípios contemplados com o programa é similar a distribuição do IDH. Ao não promover nenhuma distorção nesses níveis, houve uma distribuição subótima. O programa reproduziu a desigualdade já existente na distribuição do IDH.

No IDHB, em todos os cenários (exceto Região Sul) a distribuição do PST produziu uma distribuição conservadora. Especialmente nas regiões Norte e Nordeste, onde a perda percentual foi mais elevada, o PST piorou as condições de oferta de políticas de esporte e de lazer de vetor redistributivo. Na região Nordeste os municípios de IDHB representam $60,5 \%$ do total. Na descentralização do PST apenas 26,3\% (diferença de 34,2 pp). Ou seja, na região de maior número e percentual de municípios vulneráveis teve baixa adesão percentual dos municípios ao PST.

Em todos os cenários do IDHA houve distorção alocativa conservadora. O percentual de municípios de IDHA é menor do que o percentual de descentralização do PST nas regiões. Isso significa que os municípios de IDHA possuem certas características que garantem maior descentralização do programa. O desenho do 
programa está melhor adequado às necessidades dos municípios de maior IDH. No IDHMA também observa a mesma realidade nas regiões Sul, Sudeste e Centro-Oeste. Fator agravante à análise. Municípios de maior desenvolvimento das regiões de maior desenvolvimento econômico e social (Sul e Sudeste) conseguem melhor descentralizar o PST.

A prefeitura é a instância de Estado mais descentralizada da federação brasileira. Os gatilhos institucionais para garantir a implementação de políticas de esporte e de lazer por dentro do Estado necessitam do fortalecimento das prefeituras quanto parceiro estratégico do Governo Federal. Nesse sentido, a leitura de quais seriam os parceiros estratégico à implementação do programa também fazem parte da definição do que deve ser o programa. No que diz respeito às prefeituras (e observando estritamente os achados da pesquisa), algo está fora do lugar.

Sob essa perspectiva é possível inferir que o desenho do PST para às prefeituras (seus incentivos) produzem severas distorções alocativas e privilegiam regiões e municípios de maior desenvolvimento. Dessa forma, o Ministério do Esporte atua para impedir que o Governo Federal, na área esportiva e de lazer, cumpra o que está previsto no inciso $3^{\circ}$ do $\operatorname{artigo~} 3^{\circ}$ da Constituição Federal de 1988.

\section{Considerações Finais}

Em linhas gerais, o resultado encontrado não representa uma contradição com o modelo federalista brasileiro. A adesão voluntária ao programa por parte de prefeituras com grandes diferenças econômicas, informacionais e de quadros técnicos tende a reproduzir as desigualdades já sedimentadas na sociedade brasileira (ARRETCHE; VAZQUEZ; GOMES, 2012; ARRETCHE; MARQUES, 2007; MELO, 2005). Esses 
resultados são suportados pelo modelo federalismo brasileiro. Como revela Almeida (1995, p. 105)

[...] a descentralização em curso nas áreas sociais analisadas corre o risco de confirmar, quando não de agravar (grifo nosso), as disparidades intra e inter-regionais geradas sob o federalismo centralizado. A forma pela qual se tem processado o rearranjo de competências, especialmente nos casos de descentralização por ausência, pode neutralizar ou perverter o efeito de equidade que toda política social tem pó meta.

Apesar do desejo de garantir democratização do esporte educacional, portanto, de garantia de equidade, os incentivos do programa à descentralização não estão adequados às necessidades das regiões mais vulneráveis. Nesse sentido, encontrar uma realidade diferente, na direção de maior privilégio das cidades mais vulneráveis, seria um efeito não esperado do programa.

Talvez a observação geral mais importante seja o fato de apenas $8,9 \%$ dos municípios terem obtido algum núcleo do PST nos dez primeiros anos de sua implementação produzindo um vazio assistencial da ordem de $98,1 \%{ }^{4}$. Se levarmos em consideração que a grande maioria dos municípios teve um núcleo, pelo menos, do programa por um ou dois anos, o resultado se torna ainda mais preocupante para um programa que precisa diminuir a desigualdade na oferta desse bem social e intenciona democratizar o acesso ao esporte educacional.

A autonomia política não impõe obrigação à cooperação com o governo federal (MINHOTO, 2014; ARRETCHE, 2004). Nesse sentido, não há anomalia no resultado, apenas, talvez, a constatação de que os incentivos à descentralização do programa sejam muito tímidos e incapazes de efetivarem aquilo que está previsto no programa e garantir a diminuição da desigualdade. Diante desse cenário, torna-se importante que o PST

\footnotetext{
${ }^{4}$ Para análise do vazio assistencial ver Santos; Starepravo e Souza Neto (2015).
} 
revise seus incentivos à descentralização top-down. Quando os municípios vulneráveis perdem, os não vulneráveis ganham na descentralização do Programa Segundo Tempo.

\section{REFERÊNCIAS}

ALMEIDA, M. H. T. A política social no Governo Lula. Novos Estudos CEBRAP, São Paulo, n. 70, p. 7-17, 2004.

Federalismo e políticas sociais. Revista Brasileira de Ciências Sociais, São Paulo, v. 10, n. 28, p. 88-108, 1995.

ARRETCHE, M. Federalismo e políticas sociais no Brasil: problemas de coordenação e autonomia. São Paulo em Perspectiva, São Paulo, v. 18, n. 2, p. 17-26, 2004.

.; VAZQUEZ, D.; GOMES, S. Descentralização e autonomia: deslocando os termos do debate. In. LAVALLE, A. G. (Org.) O Horizonte da política - Questões emergentes e agendas de pesquisa. São Paulo: UNESP, 2012. p. 29-62.

.; MARQUES, E. Condicionantes locais da descentralização das políticas de saúde. In: HOCHMAN, G.; ARRETCHE, M.; MARQUES, E. (Orgs.) Políticas públicas no Brasil. Rio de Janeiro: Ed. FIOCRUZ, 2007. p. 173-204.

BONFIM, W. L. S., SILVA, I. N. Instituições políticas, cidadania e participação: a mudança social ainda é possível? Revista Sociologia Política, Curitiba. 21. p. 109-123, nov, 2003.

BRASIL. Constituição (1988). Constituição da República Federativa do Brasil. Brasília: Senado Federal: Centro Gráfico, 1988. 292 p.

Instrução Normativa n.01, de15 de janeiro de 1997. Celebração de Convênios. Disciplina a celebração de convênios de natureza financeira que tenham por objeto a execução de projetos ou realização de eventos e dá outras providências. Diário Oficial da União. Brasília, DF: Tesouro Nacional.

Portaria Interministerial $\mathrm{n}^{\mathrm{o}} 127$ de 29 de maio de 2008. Estabelece normas para execução do disposto no Decreto $n^{0}$ 6.170, de 25 de julho de 2007, que dispõe sobre as normas relativas às transferências de recursos da União mediante convênios e contratos de repasse, e dá outras providências. Disponível em: http://www.conveniosfederais.com.br/Portarias/InterMin127_08_busca_nova.html, Acesso em: 10 nov. 2017.

BUSSO, G. La vulnerabilidad social y lãs políticas sociales inícios del siglo XXI. Santiago do Chile: CEPAL/Celade, 2001. 
CASTRO, S. B. E. de. Políticas públicas para o esporte e lazer e o ciclo orçamentário brasileiro (2004-2011): prioridades e distribuição de recursos durante os processos de elaboração e execução orçamentária. 378 f. Tese (Doutorado em Educação Física) - Universidade Federal do Paraná, Curitiba, 2016.

FERREIRA, H.; CASSIOLATO, M.; GONZALEZ, R. Uma experiência de desenvolvimento metodológico para avaliação de programas: o modelo lógico do Programa Segundo Tempo. Texto para Discussão n. 1369, IPEA, 2009.

HILL, M. Implementação: uma visão geral. In. SARAVIA, E. e FERRAREZI, E. (Org.) Políticas públicas. Brasília: ENAP, 2006. p. 61-90.

IBGE - Instituto Brasileiro de Geografia e Estatística. Perfil dos Municípios Brasileiros 2006. Disponível em: http://www.ibge.gov.br . Acesso em: 10 mar. 2016.

IBGE - Instituto Brasileiro de Geografia e Estatística. Perfil dos Municípios Brasileiros 2016. Rio de Janeiro: IBGE, 2017.

MACHADO, José Angelo. Gestão de políticas públicas no Estado federativo: apostas e armadilhas. Dados-Revista de Ciências Sociais, p. 433-457, 2008.

MARQUES, E.; ARRETCHE, M. Condicionantes locais da descentralização das políticas de saúde. Caderno CRH, Salvador, n. 39, p. 55-81, jul./dez. 2003.

MELO, M. A. O sucesso inesperado das Reformas de Segunda Geração: federalismo, reformas constitucionais e política social. Dados, Rio de Janeiro, v. 48, n. 4, p. 845-889, 2005.

MENDES, Constantino Cronemberger. O território e o arranjo federativo para o desenvolvimento brasileiro: o caso do Nordeste. LINHARES, P.T.F.; MENDES, C.C.; LASSANCE, A. (Org.) Federalismo à Brasileira questões para discussão, 2012. P. 89-111.

MINHOTO, A. C. B. Federalismo, estado federalista e a revalorização do município: um novo caminho para o século XXI? Revista Brasileira de Políticas Públicas, v. 3, n. $2,2014$.

PAES, N. L.; SIQUEIRA, M. L. Desenvolvimento regional e federalismo fiscal no Brasil: em busca da igualdade na distribuição de receitas. Economia Aplicada, São Paulo, v. 12, n. 4, p. 707-742, out./dez. 2008.

SABATIER, Paul A. Top-down and bottom-up approaches to implementation research: a critical analysis and suggested synthesis. Journal of Public Policy, v. 6, n. 1, p. 21 48, 1986.

SANTOS, Edmilson Santos; STAREPRAVO, Fernando Augusto; SOUZA NETO, Marina Silva. Programa Segundo Tempo e o vazio assistencial na região Nordeste. Movimento, v. 21, n. 3, p. 759, 2015. 
SOUZA, Celina. Federalismo e gasto social no Brasil: tensões e tendências. Lua Nova, São Paulo, v. 52, p. 5-28, 2001.

\section{Endereço do Autor:}

Edmilson Santos dos Santos

Colegiado de Educação Física/UNIVASF

Rua José de Sá Maniçoba s/n - Centro

Petrolina - PE - 56.304-917

Endereço Eletrônico: edmilsonss.univasf@gmail.com 